\title{
Wi-Fi-Based Localization in Dynamic Indoor Environment Using a Dynamic Neural Network
}

\author{
Djabri Fahed and Rongke Liu
}

\begin{abstract}
Recently, there is an increasing interest in WIFI positioning systems due to the cost and availability of this technology. However, the main problem in WIFI-based localization is the severe fluctuation of received signal strength even for a static client. In this paper, we consider the localization of a wireless device using a dynamic neural network. Many types of dynamic neural networks are simulated, and then we will choose the one that gives best estimations to do real experiments. The proposed approach demonstrates significant improvements in the experiments and simulation.
\end{abstract}

Index Terms-Indoor localization, neural networks, RSS time varying, WIFI positioning systems.

\section{INTRODUCTION}

Accurate indoor geolocation is an important and novel emerging technology for commercial, public safety, and military applications. Recently, there is increasing interest in WIFI positioning systems due to the cost and availability of this technology. Wide applications of indoor localization such as location-based services applications have been emerging very fast [1], which demands a closer attention to their use. However, the main problem in WIFI-based localization is the severe fluctuation of received signal strength (RSS) even for a static client. It is not easy to model the radio propagation in the indoor environment because of severe multipath, low probability for availability of line-of-sight (LOS) path, and specific site parameters such as floor layout, moving objects, and numerous reflecting surfaces. There is no good model for indoor radio multipath characteristic so far [2]. Except using traditional triangulation, positioning algorithms using scene analysis or proximity are developed to mitigate the measurement errors. Targeting different applications or services, these three algorithms have unique advantages and disadvantages.

RF-based scene analysis refers to the type of algorithms that first collect features (fingerprints) of a scene and then estimate the location of an object by matching online measurements with the closest a priori location fingerprints. RSS-based location fingerprinting is commonly used in scene analysis. Location fingerprinting refers to techniques that match the fingerprint of some characteristic of a signal that is location dependent. There are two stages for location fingerprinting: offline stage and online stage (or run-time stage). During the offline stage, a site survey is performed in an environment. The location coordinates/labels and

Manuscript received November 23, 2012; revised January 8, 2013. This work was supported in part by the School of Electronic and Information Engineering, Beijing University of Aeronautics and Astronautics.

The Authors are with the Beijing University of Aeronautics and Astronautics, China (e-mail: fahd.zboot22@gmail.com; rongke.liu@gmail.com). respective signal strengths from nearby base stations/measuring units are collected. During the online stage, a location positioning technique uses the currently observed signal strengths and previously collected information to figure out an estimated location. The main challenge to the techniques based on location fingerprinting is that the received signal strength could be affected by diffraction, reflection, and scattering in the propagation indoor environments. There are at least five location fingerprinting-based positioning algorithms using pattern recognition technique so far: probabilistic methods [3], k-nearest-neighbor ( $\mathrm{kNN})$ [3], [4], neural networks [5]-[7], support vector machine (SVM) [8], and smallest M-vertex polygon (SMP) [9].

For any indoor positioning systems, three elements are needed and used in order to design an operational system. In this work, Wi-Fi represents the infrastructure while fingerprinting and neural networks represent the technique and pattern matching algorithm respectively.

An Artificial Neural Network is an information processing paradigm that is inspired by the way biological nervous systems, such as the brain processes information. The key element of this paradigm is the novel structure of the information processing system. It's composed of a large number of highly interconnected processing elements (neurons) working in unison to solve specific problems.

In this paper, we will try to use the features of dynamic neural networks (DNN) to get good estimation of the receiver's location.

\section{LOCALIZATION ALGORITHM WITH DYNAMIC NEURAL NETWORK (LAD)}

\section{A. Global Scheme of LAD}

We will study the ability of DNN to be used in resolving the RSS fluctuation problem in Wi-Fi localization. In other words, the DNN would make the relation between $\operatorname{RSS}(t)$ which is depending of time with position $(x, y)$ which is fixed on time but changing on space.

In Fig. 1, the localization using DNN process is presented. In offline stage, we collect RSS at $m$ known positions, from $n$ Access Points (AP), and each known position is represented by $k$ RSS samples (varying over time). Thus, we will get a dynamic database represented by the matrix $n \times m \times k$ RSS (Dynamic data base describe the distribution of received power over distance and time).

The DNN is trained by couple $(n \times m$ RSS, $2 \times m(x, y))$ with $\mathrm{k}$ delays. In online phase, when $(n \times k)$ RSS values are presented at the input, the DNN calculates k positions. Then, we take the kth estimated positions as the final estimation. 


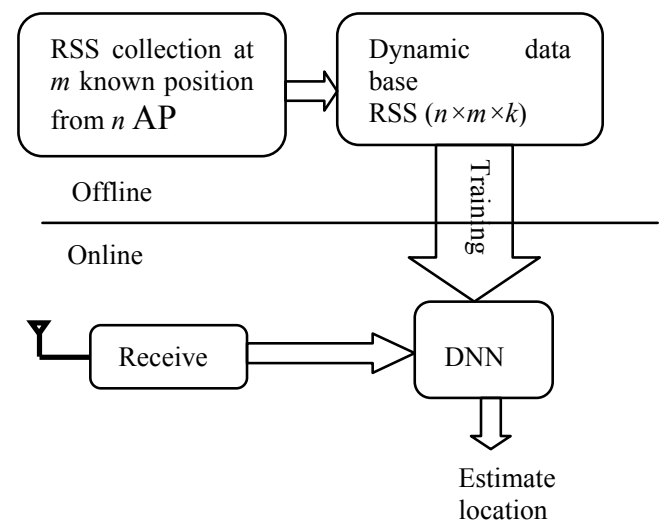

Fig. 1. Global scheme of LAD

\section{B. Generation of RF map}

For simulation, three types of point position are generated:

- AP' s positions

- Training points' position

- Testing points' position

The generated RSS is obtained using the model which is described in (1)

$$
R S S(d, t)=P_{t}-10 n \log (d)+X_{\sigma}(t)
$$

$P_{t}:$ Transmitted power

$n$ : Path loss exponent

$d$ : distance between the receiver and transmitter

$X_{\sigma}$ : Random variable following Normal distribution $(0, \sigma)$ and it represents noise.

$\sigma:$ Environment deviation

Two kinds of RSS are generated: stationary and non-stationary RSS. The non-stationary is introduced in this work because it has shown in [10] that the distribution of the RSS is not usually Gaussian, it is often left-skewed and the standard deviation varies according to the signal level. Also, it has found that the RSS is stationary under certain circumstances, but in general, such a conclusion cannot be made. Therefore, the non-stationary aspect should be taken in consideration which wasn't taken in previous works.

The non-stationarity of any given time series data (in our case is RSS) means that the moments (i.e. Mean and variance) of the system are not static in time. Basing on this definition of non stationarity [11] the non-stationary RSS is generated using (1) with time-variant deviation. Fig. 2 shows the two types of RSS at a précised point.

\section{Selection and Designing of the DNN}

Three kinds of DNNs can be used (Fig. 3):

- NARX : Nonlinear Autoregressive Network with eXogenous

- FFTD : Feed Forward Time-Delay Neural Network

- LRN : Layer-Recurrent Network

They all have the same multi-layer structure (one hidden layer with one neuron, the transfer functions are set to pureline).

DNN generates two kinds of errors: Estimation Error over time represented by $e(t)$ and Estimation Error over space represented by $e(d)$.

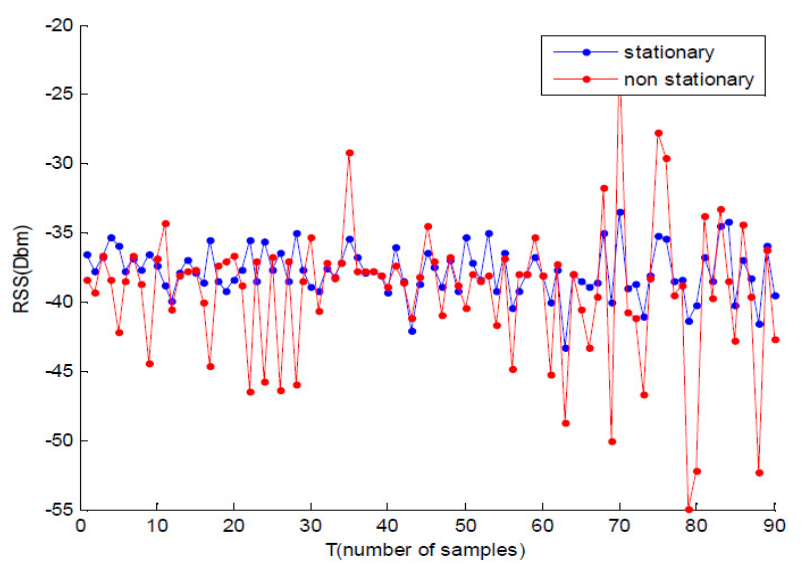

Fig. 2. Stationary and non stationary RSS at $(\mathrm{x}, \mathrm{y})$

Firstly, the evolution of $e(t)$ of the three DNN is checked out. For that, DNNs are trained by the same matrices. Then, we calculate the mean square error (MSE) over time of one testing point (variation over space is fixed) and the error is described as following:

$$
e(k)=x_{\text {test }}-\hat{x}(k) \quad \text { With } k=12 \ldots . . T
$$

$T$ is the length of RSS vector of one testing position.

Secondly, the performance of the three networks is tested with many testing points (more than one) to find out the estimation error over space. The output over time of each testing position is taken at $t=T$ for networks with feedback (LRN and NARX) and the mean of $e(t)$ for FFTD (because has no feedback).

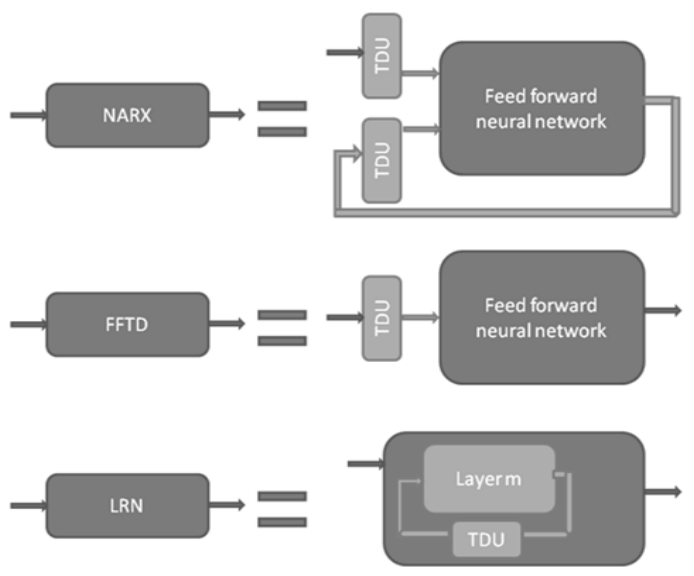

Fig. 3. Types of DNNs

TDU is a time delay unit

The selection is based on minimizing the couple $(e(t)$, $\mathrm{MDE})$. MDE is the mean distance error given in (3).

$$
M D E=\frac{\sum_{i=1}^{N} \sqrt{\left(x_{i}-\hat{x_{i}}\right)^{2}+\left(y_{i}-\hat{y_{i}}\right)^{2}}}{N}
$$

$N$ is the number of points

The cumulative probability functions (CDF) of the distance error is used for measuring the precision of a system. When two positioning techniques are compared, if their 
accuracies are the same, we prefer the system with the CDF graph, which reaches high probability values faster, because its distance error is concentrated in small values [1].

Table I gives the simulation parameters.

TABLE I: SIMULATION PARAMETERS OF DNN'S ERROR ANALYSIS

\begin{tabular}{|l|l|}
\hline \multicolumn{2}{|c|}{ Environment } \\
\hline$\sigma$ & 2 \\
\hline Path loss exponent $n$ & 2 \\
\hline Number of AP & 3 \\
\hline
\end{tabular}

\begin{tabular}{|l|l|}
\hline \multicolumn{2}{|c|}{ Fingerprinting } \\
\hline Training sets & 100 \\
\hline Testing sets & 10 \\
\hline RSS samples per location & 60 \\
\hline
\end{tabular}

Fig. 4 shows the evolution of DNNs' MSE over time of the coordinate $x$ of one test point. The MSE of NARX is considerably increasing in time while FFTD and LRN's MSE is fluctuant around a mean. Those phenomena are due to the feedback and its position as well. For NARX, the presence of the feedback from output to input (which includes two layers) creates an accumulation of the error over time because the position is static in time but for FFTD the output is strictly following the input evolution. For LRN, the error of the first layer is pursuing the input variation but this error is reduced after the second layer.

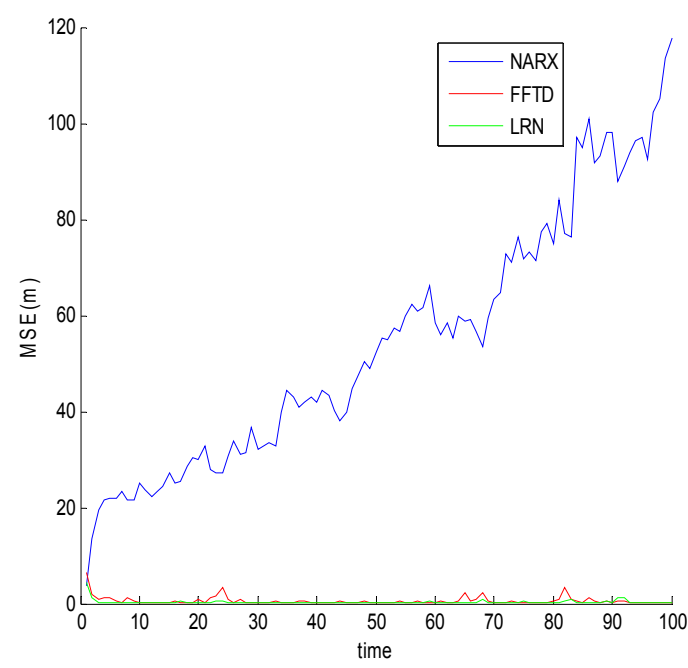

Fig. 4. MSE's evolution over time of coordinate $\mathrm{x}$ for (NARX, LRN,FFTD)

TABLE II: MDE AND CDF OF NARX, LRN AND FFTD
\begin{tabular}{|l|l|l|l|}
\hline & FFTD & LRN & NARX \\
\hline MDE (m) & 1.673 & 3.729 & 6.534 \\
\hline CDF at 4m & $91.40 \%$ & $55.58 \%$ & $9.26 \%$ \\
\hline
\end{tabular}

Those results are not conclusive, because we still have to choose between FFTD and LRN. For that, we need to calculate the CDF of NARX, LRN and FFTD estimation errors over distance. Table II demonstrates that FFTD is obviously showing better estimations. Moreover, the time of training of FFTD is largely less than NARX's and LRN's time.

\section{Selected DNN's Conception}

The FFTD will have three layers and each layer has a different tasks:

- Input layer: the role of this layer is to cluster the variation of RSS over time of each position. The transfer function will be selected among these three functions: 'tribas', 'radbas' and 'tangsig'.
- Hidden layer: is considered as a nonlinear filter of the RSS fluctuation over time, contains $m$ neurons with 'logsing' as transfer function which is saturation function. Thus, in online phase the last estimation in time is taken instead of averaging all estimations.

- Output layer: contains 2 neurons of 'purelin' activation function.

\section{Simulation AND EXPERIMENTAL RESUlts}

\section{A. Simulation Results}

From [12], [13], three kinds of typical environment have been selected to study the performance of the proposed approaches and those values (presented in Table III) are empirical which make the simulation more realistic. Two kinds of RSS are used for the simulation stationary and non-stationary in order to carry out the impact of the non-stationarity on the localization performances.

It is apparent from the table IV that the proposed approach is giving good estimations. However, the ameliorations which have been given in term of MDE and CDF aren't constantly the same for the different environments. That dissimilarity is occasioned by the design of the DNN (items selection and optimization). Furthermore, this approach reduces the effect of the non stationarity on localization errors and the results of stationary and non-stationary RSS are almost the same.

\begin{tabular}{|c|l|c|c|}
\hline \multicolumn{1}{|c|}{ TABLE III: TYPICAL ENVIRONMENT } & \multicolumn{1}{|c|}{ Description } & n & $\boldsymbol{\sigma}$ \\
\hline Environment & \multicolumn{1}{|c|}{ (indoor building with Line Of sight } & 1.8 & 1.89 \\
\hline$\# 2$ & $\begin{array}{l}\text { indoor building with Soft cubicle partitions } \\
\text { (metals) }\end{array}$ & 3.5 & 4.62 \\
\hline$\# 3$ & Obstruction in building (bricks) & 6 & 5.23 \\
\hline
\end{tabular}

TABLE IV: LOCALIZATION PERFORMANCE FOR TYPICAL ENVIRONMENTS

\begin{tabular}{|l|l|c|c|}
\cline { 3 - 4 } \multicolumn{2}{|c|}{$\# 1$} & Stationary & Non Stationary \\
\cline { 2 - 4 } & CDF at 2m (\%) & 89.24 & 1.11 \\
\hline \multirow{2}{*}{$\# \mathbf{2}$} & MDE(m) & 1.17 & 0.82 \\
\cline { 2 - 4 } & CDF at 2m (\%) & 82.86 & 99.47 \\
\hline \multirow{2}{*}{$\# \mathbf{3}$} & CDF at 2m (\%) & 89.28 & 0.88 \\
\cline { 2 - 4 } & MDE(m) & 0.96 & 99.94 \\
\hline
\end{tabular}

\section{B. Experimental Results}

A realistic RSS data is collected in a residential apartment which the plan is presented in figure 5 . The dimension of the environment is $7.8 \times 13.7$ meters. Every location in this environment is covered by one IEEE $802.11 \mathrm{n}$ AP on average. We adopt two kinds of receivers: Lenovo X60 laptop with a Windows XP operating system and ZTE U880 mobile phone with android 2.2.1 operating system. Netstembler is used by the laptop to pick up the signal and every sample is taken within 1 second while WiGLE is used by the mobile phone and every sample is sensed within 2.6 second, both are free software. 
In the target indoor environment, 113 samples of RSS by laptop and 60 samples by mobile phone at 47 locations to build the training database. Then, 5 test points will be collected in this area. In order to test the robustness of the positioning system, only 60 samples of RSS are taken by the two receivers for each test location. It's important to note that the receiver's orientation has kept the same direction (north) in order to not interfere with impact of orientation on accuracy and robustness of the system [10].

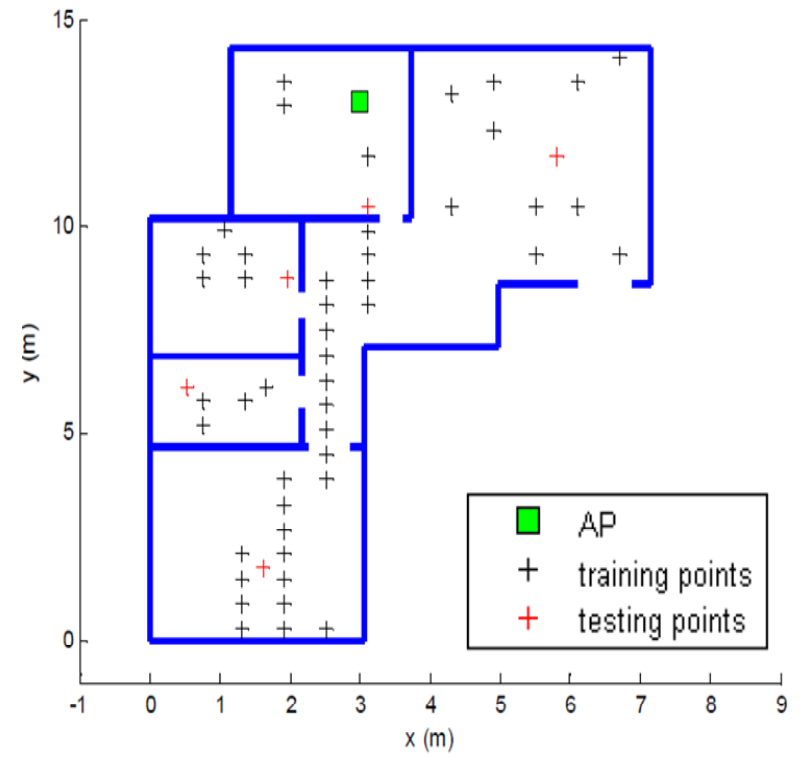

Fig. 5. Studied environment

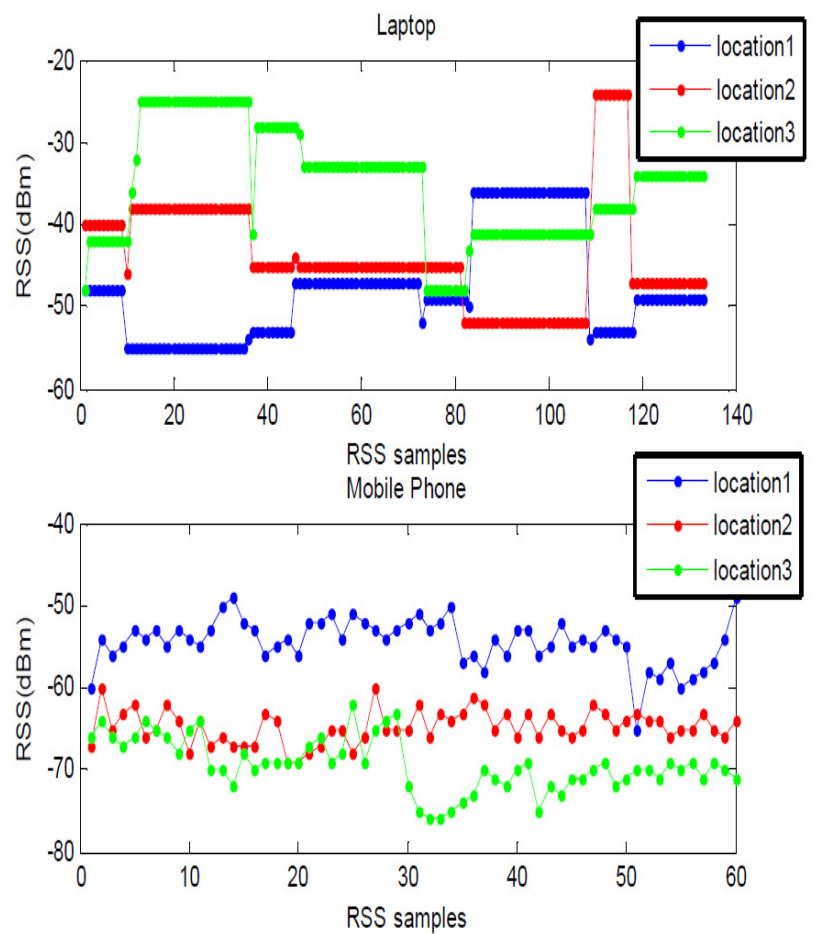

Fig. 6. RSS of mobile phone and laptop at the same locations

The Fig. 6 shows the variation of RSS of mobile phone and laptop in time at several locations. Firstly, the way of RSS varies in time at those locations is dissimilar for both receivers (mobile phone and laptop) and it is the consequence of environment's dynamism. Secondly, the difference of RSS wave form between the two receivers at the same location is due to two reasons: The sensing time is different, for the mobile phone is much longer than laptop's time, and the characteristic of antenna's receiver.

Table $\mathrm{V}$ shows the mean distance error and cumulative probability function calculated with RSS samples in figure 6 .

The difference in performances between using laptop and mobile phone is due to the wave form and nature of the measured RSS.

We can see that experimental results are not very different from those of the simulation. Those differences can be explained by a lot of reasons: difference between the environment parameters in the simulation and the experimentation, accuracy of the measured RSS, learning process.

TABLE V: EXPERIMENTAL MDE AND CDF

\begin{tabular}{|l|c|c|}
\cline { 2 - 3 } \multicolumn{1}{c|}{} & Laptop & Mobile \\
\hline MDE(m) & 1.86 & 1.93 \\
\hline CDF at 4m (\%) & 90.13 & 92.66 \\
\hline
\end{tabular}

\section{CONCLUSION}

The use of dynamic neural network in geolocation may reveal interesting. The proposed approach with its schemes of optimization has been tested under three typical environments and the results showed that our solution gives good results. Moreover, the impact of non stationarity of RSS has been demonstrated and we can see that that the DNN reduces its effect on localization performance.

\section{REFERENCES}

[1] F. Sallabi, G. Ditsa, E. Barka, and H. El-Khatib, "Business model for emerging real-time location-based services: A technical perspective," in Proceedings of the 2010 International Conference on Wireless Information Networks and Systems (WINSYS), July 2010.

[2] K. Pahlavan, X. R. Li, and J. P. Makela, "Indoor geolocation science and technology," IEEE Communications Magazine, vol.40, Issue.2, Feb. 2002.

[3] B. Dawes and K. W. Chin, "A comparison of deterministic and probabilistic methods for indoor localization," Journal of Systems and Software, vol. 84, issue 3, March 2011.

[4] C.W. Yuen, N. Balasubramaniam, and N. Md. Din, "Improvement of indoor location sensing algorithm using wireless local area network (WLAN) IEEE 802.11b, " in Proceedings of the IEEE 9th Malaysia International Conference on Communications, Kuala Lumpur, Malaysia, 2009.

[5] A. Shareef, Y. F. Zhu, and M. Musavi, "Localization using neural networks in wireless sensor networks," Mobilware'08, February 12-15, 2008 .

[6] U. Ahmed, A. Gavrilov, U. Nasir, M. Iqbal, S. J. Cho, and S. Y. Lee, "In-building localization using neural networks," in IEEE International Conference on Engineering of Intelligent Systems, 2006.

[7] A. Taok, N. Kandil, and S. Affes, "Neural networks for fingerprinting-based indoor localization using ultra-wideband," IEEE Journal of Communications, vol. 4, no. 4, May 2009.

[8] M. Brunato and R. Battiti, "Statistical learning theory for location fingerprinting in wireless LANs", Computer Networks, vol. 47, issue 6, pp. 825-845, April 2005.

[9] P. Prasithsangaree, P. Krishnamurthy, and P.K. Chrysanthis, "On indoor position location with wireless LANs", The 13th IEEE International Symposium on Personal, Indoor and Mobile Radio Communications, 2002.

[10] K. Kaemarungsi and P. Krishnamurthy, "Properties of indoor received signal strength for WlAN location fingerprinting," in Proceedings of the First Annual International Conference on Mobile and Ubiquitous Systems: Networking and Services (MobiQuitous'04), 2004. 
[11] M. Butler and D. Kazakov, "The effects of variable stationarity in a financial time-series on artificial neural networks," in IEEE Symposium on Computational Intelligence for Financial Engineering and Economics (CIFEr), 2011

[12] S. Y. Yeong, A. S. Wafaa, and T. C. Wan, "Indoor WLAN monitoring and planning using empirical and theoretical propagation models," in Second International Conference on Network Applications Protocols and Services (NETAPPS), 2010

[13] T. Sadiki and P. Paimblanc, "Modeling new indoor propagation models for WLAN based on empirical results," in 11th International Conference on Computer Modeling and Simulation, 2009

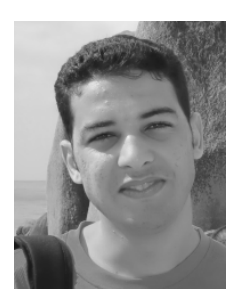

Djabri Fahed was born in Algeria on February 2, 1985. He received the preparatory engineering degree in 2005 . He earned the engineer degree in electrical engineering in 2008 from Polytechnic School. He is currently working toward the Ph.D. degree in electrical engineering from Beihang University. His research interests include neural networks and geolocalization.

Rongke Liu received the B.S. and Ph.D. degree in electrical engineering from Beihang University in 1996 and 2002, respectively.

He was an associate professor from 2004 to 2010 with the department of electrical engineering, Beihang University. Since 2010, he has been with the school of electronics and information engineering, Beihang University, where he is a full professor. His research interests include space communication, wireless multimedia communication and 3D TV. 